\title{
Effect of Cosmological Constant on Amplitude Variation of Boson Star
}

\author{
Bharti Jarwal $^{1 *}$ and S. Somorendro Singh ${ }^{2 *}$ \\ ${ }^{1,2}$ Department of Physics and Astrophysics, University of Delhi, Delhi-110007, India \\ *Corresponding Author: sssingh@physics.du.ac.in
}

Available online at: www.isroset.org

Received: 28/Mar/2020, Accepted: 21/Apr/2020, Online: 30/Apr/2020

\begin{abstract}
The effect of cosmological parameter on rotation of boson star oscillations is studied. The study found that the amplitude of rotation of boson star ground state and excited state decreased under the correction of cosmological constant in the Lagrangian density of Boson star.
\end{abstract}

Keywords- compact objects, boson star, QGP, cosmological constant

\section{Introduction}

In recent years, scientists have discovered that the expansion rate of the Universe is increasing rather than decreasing [1,2]. The cosmic acceleration leads to an additional term in Einstein's field equation which is known as the cosmological constant. The cosmological constant, the value of the energy density of the vacuum of space, is the simplest form of dark energy and it provides a good fit to many cosmological observations. A positive vacuum energy density resulting from a positive cosmological constant (implying a negative) pressure gives an accelerated expansion of the Universe consistent with the observations. Albert Einstein originally introduced this term in 1916 as a modification of his field equation to achieve a stationary Universe [3], but quickly abandoned the concept after Hubble's discovery of the Universe expanding [4]. The work explores the consequences of a non-vanishing cosmological constant for spherically symmetric mass distributions of compact stars such as quark or neutron stars. Neutron stars (NSs) and black holes (BHs) are among the most exotic objects produced in nature. They are formed in the core collapse of massive stars like supernova and, in many cases, their formation is associated with powerful astrophysical transients such as supernovae and gammaray bursts. The first calculations of neutron star models were performed by Oppenheimer and his collaborators [6], who assumed neutron star matter to be composed of an ideal gas of free neutrons at high density. By studying the masses of these objects we can better understand their formation process and associated explosions. In addition, accurate measurements of masses of NSs and BHs provide essential input to our understanding of a wide range of astrophysical phenomena produced by these objects, from gravitational waves formed in compact object mergers to X-ray binaries. Therefore the studies of neutron stars in Einstein- $\Lambda$ gravity and the effect of cosmological constant effect are taken into account in the study of giant objects [9]. The paper explores these different solutions, in particular the consequences of a non-vanishing cosmological constant for compact objects specifically boson star. Studying these implications can yield great insight into the evolution of compact objects. The boson stars are very fascinating objects as their self gravity is not balanced by the degeneracy pressure like the other compact stars such as a white dwarf or a neutron star, but the Heisenberg uncertainty principle plays a crucial role in their stability [8]. Boson stars and boson shells are hypothetical astronomical objects consisting of bosons. They could possibly be detected by gravitational radiation emitted, for example, by a pair of co- orbiting boson stars [5, 11], and could possibly have been formed through gravitational collapse during the primordial stages of the big bang [7]. The investigation of these objects coupled to gauge field and gravity determine its theoretical existence [29]. 
They have also been proposed as dark matter candidates [12] and to predict the high density nature of universe expansion. Further, just like super-massive black holes, they could even exist in the center of galaxies [13] and could possibly explain many of the observed properties of the active galactic core [14].The study of boson shells and boson stars in scalar electrodynamics with a self-interacting complex scalar field $\Phi$ coupled to Einstein gravity in three-space onetime dimensions is of very wide ranging interest [17-23]. Kleihaus, Kunz, Laemmerzahl and List $[15,16]$ have recently studied boson shells harboring black holes and boson stars in scalar electrodynamics coupled to Einstein gravity in three-space one-time dimensions in a V-shaped scalar potential $V\left(\Phi \Phi^{*}\right) \equiv V(|\Phi|)=\lambda|\Phi|$ (where $\lambda$ is a constant) $[15,16]$. They found that the boson stars come in two types, with either ball-like or shell-like charge density $[15,16]$. They also studied the properties of these solutions and determined their domains of existence $[15,16]$. So to predict such properties and their domain of existence, we study the rotation of boson star in Newtonian approximation from which we can indirectly predict the existence and formation of these objects in our present universe[24]. To enhance the evidence many investigations are under process under the same gravity limit. The study indicates the oscillations of boson stars with different potential in $[25,26,30]$. In this paper the effect of vacuum energy density on oscillations of boson star is investigated.

\section{Newtonian Treatment for Boson Stars}

BS is considered to be a complex scalar field coupled to gravity [28]. The action principle of such system is discussed in detail under weak field approximation. The Lagrangian density of the $\mathrm{BS}$ with cosmological constant $\Lambda$ is given by

$$
£=\frac{R-2 \Lambda}{16 \pi G}+g^{\mu \nu} \partial_{\mu} \Phi^{*} \partial_{\nu} \Phi-M^{2} \Phi^{*} \Phi
$$

The metric $g^{\mu \nu}$ is expanded as follows $g^{\mu \nu}=\eta^{\mu \nu}+h^{\mu \nu}$ with $\left|h^{\mu \nu} \ll 1\right|$ and $\eta^{\mu \nu}=$ $\operatorname{diag}\left(1,-1,-r^{2},-r^{2} \sin \theta^{2}\right)$. Solving for $\Phi$ through Euler Lagrange equation of motion gives the equation of motion (EOM) of $\Phi$ as follows

$$
\square \Phi+M^{2} \Phi=0
$$

This is one EOM obtained from the Lagrangian density $£$. Further solving for $\Phi$, it is obtained as stationary solution for $\phi$, as dependence on $\mathrm{t}$ and $r$ only. So the solution is given as

$$
\Phi(\vec{r}, t)=\phi(r, \theta) e^{i \omega t} e^{i m \varphi}
$$

The another equation of motion is given as

$$
\square h_{\mu \nu}=-16 \pi G S_{\mu \nu}-\Lambda g_{\mu \nu}
$$

where $S_{\mu \nu}=T_{\mu \nu}-\frac{1}{2} \eta_{\mu \nu} T$

$$
T_{\mu \nu}=\partial_{\mu} \Phi^{*} \partial_{\nu} \Phi+\partial_{\nu} \Phi^{*} \partial_{\mu} \Phi-\eta_{\mu \nu}\left[\eta^{\alpha \beta} \partial_{\alpha} \Phi^{*} \partial_{\beta} \Phi-\left(M^{2}\right) \Phi^{*} \Phi\right]
$$

Using the weak field approximation of general relativity [27], and knowing the only relevant component of $h_{\mu \nu}$ it is found that $h_{o o}=2 V(r, \theta)$, in which $V(r, \theta)$ is Newtonian potential. In non relativistic limit, the gravitational binding energy $E$ per particle must be much smaller than M. However the scalar field frequency can be written as $w=E+M$ with $|E|<<M$. 
Considering all these arguments, the addition of cosmological constant to Lagrangian density finally leads to following equations for $\mathrm{V}$ and $\phi$ as

$$
\begin{aligned}
& \frac{-1}{2 M} \vec{\nabla}^{2} \phi+M V \phi=E \phi \\
& \vec{\nabla}^{2} V=8 \pi G M^{2} \phi-\Lambda
\end{aligned}
$$

The gauge in-variance of complex scalar field implies the conservation of the conserved current such as $j^{\mu}=i\left(\partial^{\mu} \phi \phi^{*}-\phi \partial^{\mu} \phi^{*}\right)$ with conserved particle number: $N=2 M \int \phi^{2} r^{2} \sin (\theta) d r d \theta d \varphi$. The stationary solutions of the above equations with non vanishing angular momentum can be obtained by using associated Legendre function and orthogonality relation of $P_{l}^{m}(\theta)$. So the general solutions for the above equations are obtained as below:

$$
\begin{aligned}
& \phi(r, \theta)=\frac{1}{\sqrt{4 \pi}} \sum_{l=m}^{\infty} R_{l}(r) P_{l}^{m}(\theta) \\
& V(r, \theta)=\sum_{l=0}^{\infty} V_{l}(r) P_{l}(\theta),
\end{aligned}
$$

Looking the solution it can be written as coupled equation of radial as well as angular solution. Then from the eqn.9 in which the potential function $V(r, \theta)$ is also a couple equation of $V_{l}(r)$ and $P_{l}(\theta)$. The potential $V_{l}(r)$ can be taken as $V_{\text {old }}(r)$ for the sake of convenience as taken in our earlier paper. The most generalized form of equations for $\mathrm{V}$ and $\mathrm{R}$ using associated Legendre polynomials are solved as follows,

$$
\begin{gathered}
V_{l_{0}}{ }^{\prime \prime}+\frac{2}{r} V_{l_{0}}{ }^{\prime}-\frac{l_{0}\left(l_{0}+1\right)}{r^{2}} V_{l_{0}}=G M^{2}\left(2 l_{0}+1\right) \sum_{l l^{\prime}} A_{l l^{\prime} l_{0}} R_{l} R_{l^{\prime}}-\Lambda \\
\frac{1}{2 M}\left(R_{l_{0}}{ }^{\prime \prime}+\frac{2}{r} R_{l_{0}}{ }^{\prime}-\frac{l_{0}\left(l_{0}+1\right)}{r^{2}} R_{l_{0}}\right)+2 M E R_{l_{0}}=M \frac{\left(2 l_{0}+1\right)\left(l_{0}-m\right) !}{2\left(l_{0}+m\right) !} \sum_{l=m}^{\infty} \sum_{l^{\prime}=m^{\prime}}^{\infty} A_{l l^{\prime} l_{0}} R_{l} V_{l^{\prime}}
\end{gathered}
$$

where prime denotes derivative with respect to $r$ and

$$
A_{l l^{\prime} l_{0}}=\int d x P_{l}^{m}(x) P_{l^{\prime}}^{m}(x) P_{l_{0}}
$$

The expressions are tidy to solve for the present scale. So to make the expression easy we rescale as required for the solutions. The re-scaling is done in the such way that it exactly follows the ref [10]. The typical value of cosmological parameter $\Lambda$ is taken around 0.020. Then the corresponding mass of mini BS is found to be $30 \mathrm{GeV}$. Finally this also can be re-scaled in terms of parameters of followings:

$$
\begin{aligned}
& \hat{r}=r \hat{N} M, \\
& \hat{V}(\hat{r}, \theta)=\frac{V(r, \theta)}{\hat{N}^{2}} \\
& \hat{R}(\hat{r})=\frac{R(r)(2 G)^{1 / 2}}{\hat{N}^{2}},
\end{aligned}
$$




$$
\begin{aligned}
& \hat{E}=\frac{E}{M \hat{N}^{2}} \\
& \hat{N}=G M^{2} N \frac{(2 l+1)(l-m) !}{(l+m) !} \\
& \hat{\Lambda}=\frac{\Lambda}{M^{2} N^{2}}
\end{aligned}
$$

In terms of the above parameters the set of differential equations are obtained for ground and excited states. To solve easily the above equations we take the normalization condition as:

$$
\int R_{l_{0}}^{2}(r) r^{2} d r=1
$$

For ground state $l=0$ and $m=0$ the equations for potential and radial functions are given depending on the perturbation of potential function. It is obtained as:

$$
\begin{aligned}
& \hat{V}_{0}^{\prime \prime}+\frac{2}{\hat{r}} \hat{V}_{0}^{\prime}=\hat{R}_{0}^{2}-\hat{\Lambda}, \\
& \frac{1}{2}\left[\hat{R}_{0}^{\prime \prime}+\frac{2}{\hat{r}} \hat{R}_{0}{ }^{\prime}\right]+\hat{R}_{0} \hat{E}_{0}=\hat{R}_{0} \hat{V}_{0} .
\end{aligned}
$$

The first excited state has obtained a degeneracy. The degeneracy state having $l=1, m=0$ gives the equations for potential and radial functions as:

$$
\begin{aligned}
& \hat{V}_{0}^{\prime \prime}+\frac{2}{\hat{r}} \hat{V}_{0}^{\prime}=\frac{2}{3} \hat{R}_{1}^{2}-\hat{\Lambda} \\
& \hat{V}_{2}^{\prime \prime}+\frac{2}{\hat{r}} \hat{V}_{2}^{\prime}-\frac{6}{\hat{r}^{2}} \hat{V}_{2}=\frac{2}{3} \hat{R}_{1}^{2} \\
& \frac{1}{2}\left[\hat{R}_{1}^{\prime \prime}+\frac{2}{\hat{r}} \hat{R}_{1}^{\prime}-\frac{2}{\hat{r}^{2}} \hat{R}_{1}\right]+\hat{R}_{0} \hat{E}_{10}=\hat{R}_{1} \hat{W}_{10}
\end{aligned}
$$

where $\hat{W}_{10}=\hat{V}_{0}+\frac{2}{5} \hat{V}_{2}$.

Again the first excited state having $l=1, m=1$ the degeneracy equations are obtained for potential and radial wave function as:

$$
\begin{aligned}
& \hat{V}_{0}^{\prime \prime}+\frac{2}{\hat{r}} \hat{V}_{0}^{\prime}=\frac{2}{3} \hat{R}_{1}^{2}-\hat{\Lambda} \\
& \hat{V}_{2}^{\prime \prime}+\frac{2}{\hat{r}} \hat{V}_{2}^{\prime}-\frac{6}{\hat{r}^{2}} \hat{V}_{2}=-\frac{2}{3} \hat{R}_{1}^{2}-\hat{\Lambda} \\
& \frac{1}{2}\left[\hat{R}_{1}^{\prime \prime}+\frac{2}{\hat{r}} \hat{R}_{1}^{\prime}-\frac{2}{\hat{r}^{2}} \hat{R}_{1}\right]+\hat{R}_{0} \hat{E}_{11}=\hat{R}_{1} \hat{W}_{11}
\end{aligned}
$$

where $\hat{W}_{11}=\hat{V}_{0}-\frac{1}{5} \hat{V}_{2}$.

Again the second excited state having $l=2, m=0$ the degeneracy equations are similarly obtained as:

$$
\begin{aligned}
& \hat{V}_{0}^{\prime \prime}+\frac{2}{\hat{r}} \hat{V}_{0}^{\prime}=\frac{1}{5} \hat{R}_{2}^{2}-\hat{\Lambda} \\
& \hat{V}_{2}^{\prime \prime}+\frac{2}{\hat{r}} \hat{V}_{2}^{\prime}-\frac{6}{\hat{r}^{2}} \hat{V}_{2}-\frac{-6 V(\hat{r})}{\hat{r}^{2}}=\frac{2}{7} \hat{R}_{2}{ }^{2}-\hat{\Lambda}
\end{aligned}
$$




$$
\begin{aligned}
& \hat{V}_{4}^{\prime \prime}+\frac{2}{\hat{r}} \hat{V}_{4}^{\prime}-\frac{20}{\hat{r}^{2}} \hat{V}_{4}-\frac{20 V(\hat{r})}{\hat{r}^{2}}=\frac{18}{35} \hat{R}_{2}^{2}-\hat{\Lambda} \\
& \frac{1}{2}\left[\hat{R}_{2}^{\prime \prime}+\frac{2}{\hat{r}} \hat{R}_{2}^{\prime}-\frac{6}{\hat{r}^{2}} \hat{R}_{2}\right]+\hat{R}_{2} \hat{\widetilde{E}}_{20}=\hat{R}_{2} \hat{W}_{20}+\frac{11}{7} V(\hat{r}) \hat{R}_{2}
\end{aligned}
$$

where $\hat{W}_{20}=\hat{V}_{0}+\frac{2}{7} \hat{V}_{2}+\frac{2}{7} \hat{V}_{4}$. These types of equations can be obtained for remaining degeneracy values $m=1,2$. They can also be expressed as differential equations in terms of potential and radial functions.

\section{Result and discussion}

The plots for ground, first excited and second excited state are shown in figures. The Fig.1 shows the potential presentation after introducing the cosmological constant $\Lambda$ in Lagrangian density. The potential after introducing the cosmological constant attains a sudden increase like the potential without cosmological parameter for early initial displacement and attains a constant potential for increasing displacement. The behavior is same and differs only in the strength of the potential with and without cosmological constant. The decrease in the strength of potential may be considered as due to the presence of unknown objects in the cosmological parameter which may absorb the energy of the boson star. Its maximum value is 0.00996 .

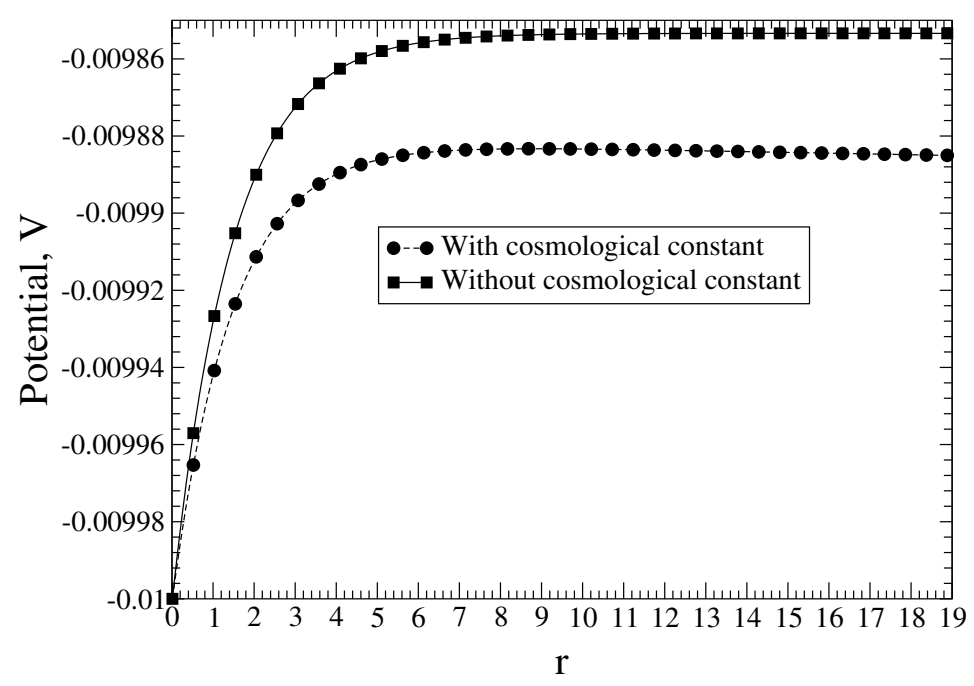

Figure 1: Potential V with and without cosmological parameter

Fig. 2 shows the radial wave function of ground state. The plot which has larger amplitude is radial wave without the cosmological constant whereas the other plot with lower amplitude is with the incarnation of cosmological constant $\Lambda$. The figure is of similar type of declining amplitude with respect to radial wave function $R$ without $\Lambda$. The value of $\Lambda$ used is 0.020 .

In Fig.3 it shows the first excited state radial wave function of two degeneracy factor of $m=0$ and 1 after the introduction of cosmological constant in Lagrangian density. Even though they have same energy, their radial wave functions have different amplitude where $m=0$ and $l=1$ has higher amplitude than $m=1$ and $l=1$. The degeneracy state i.e. $l=1, m=0$ has higher amplitude than degeneracy state i.e. $l=1, m=1$. 


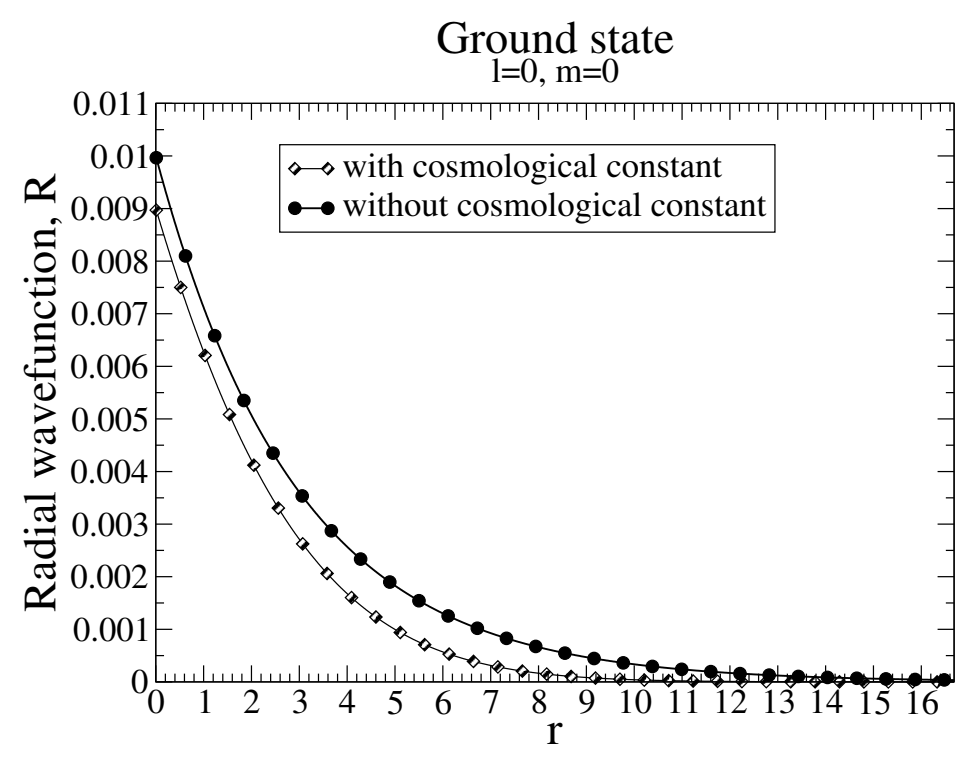

Figure 2: Radial wave function $\mathrm{R}$ of ground state with and without cosmological parameter

First excited state

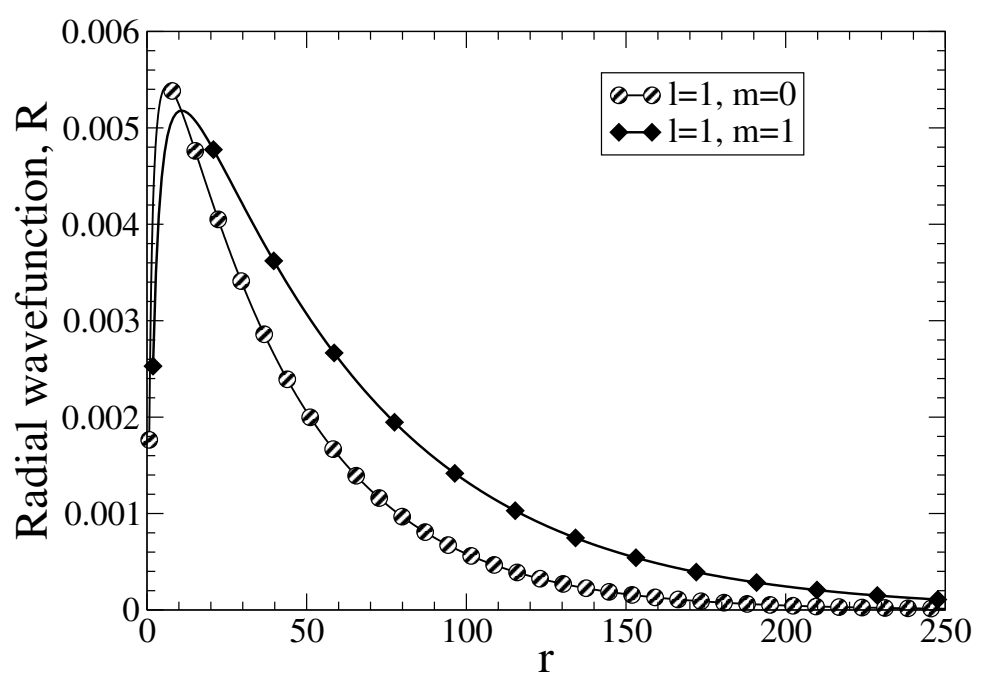

Figure 3: Effect of cosmological constant on radial wave function $\mathrm{R}$ of first excited state 
First excited state

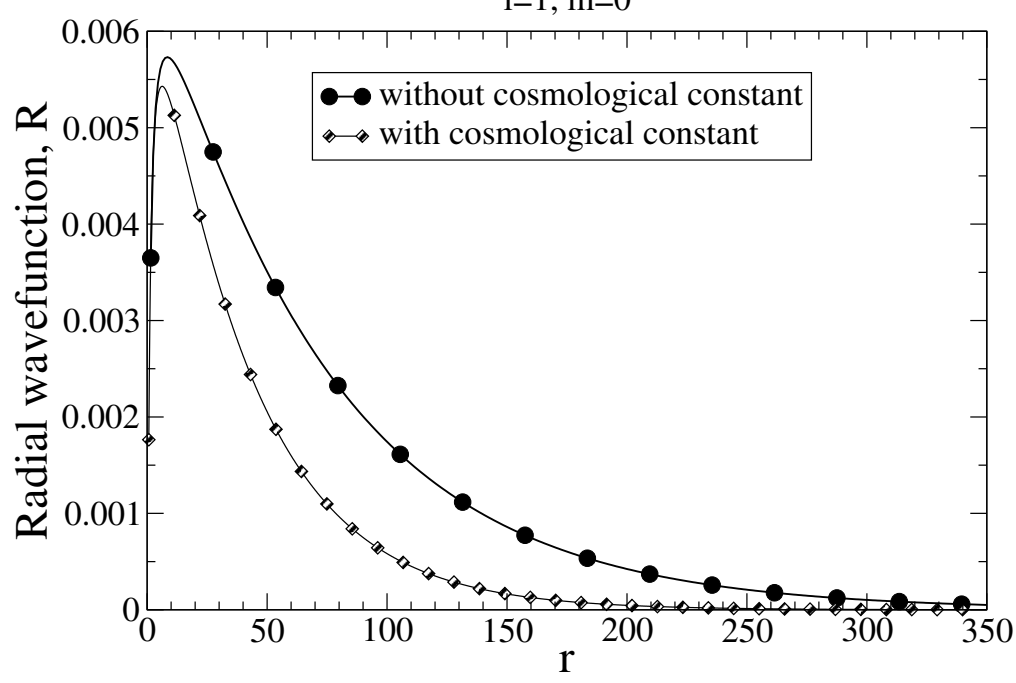

Figure 4: Radial wave function $\mathrm{R}$ of first excited state without and with cosmological constant for $\mathrm{l}=1$ and $\mathrm{m}=0$

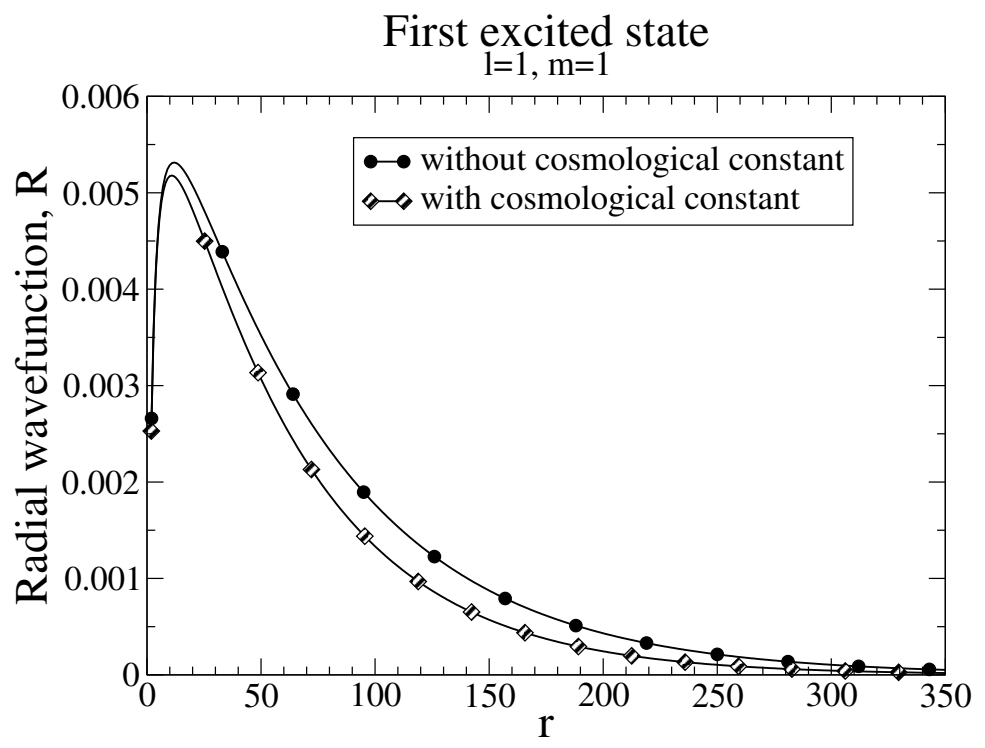

Figure 5: Radial wave function $\mathrm{R}$ of first excited state without and with cosmological constant for $\mathrm{l}=1$ and $\mathrm{m}=1$ 


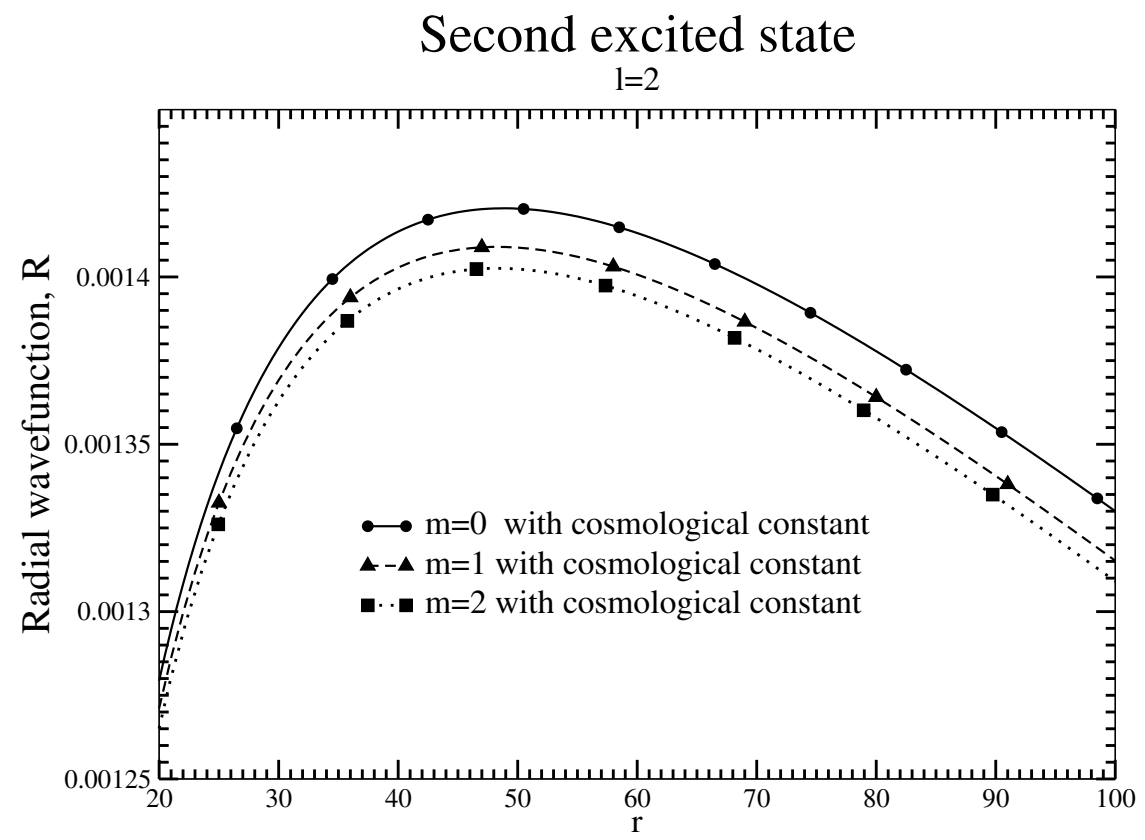

Figure 6: Radial wave function $\mathrm{R}$ of second excited state with cosmological constant for $\mathrm{l}=2$, $\mathrm{m}=0,1,2$

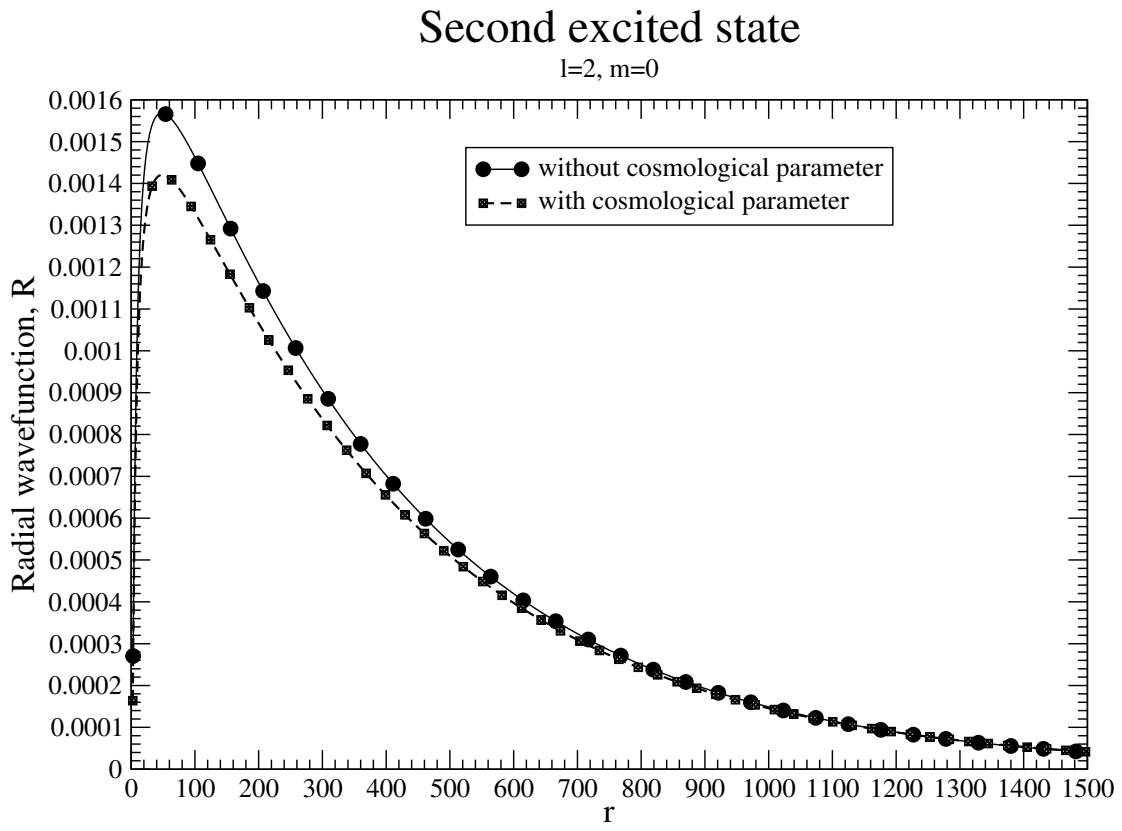

Figure 7: Radial wave function $\mathrm{R}$ of second excited state without and with cosmological constant for $\mathrm{l}=2, \mathrm{~m}=0$ 


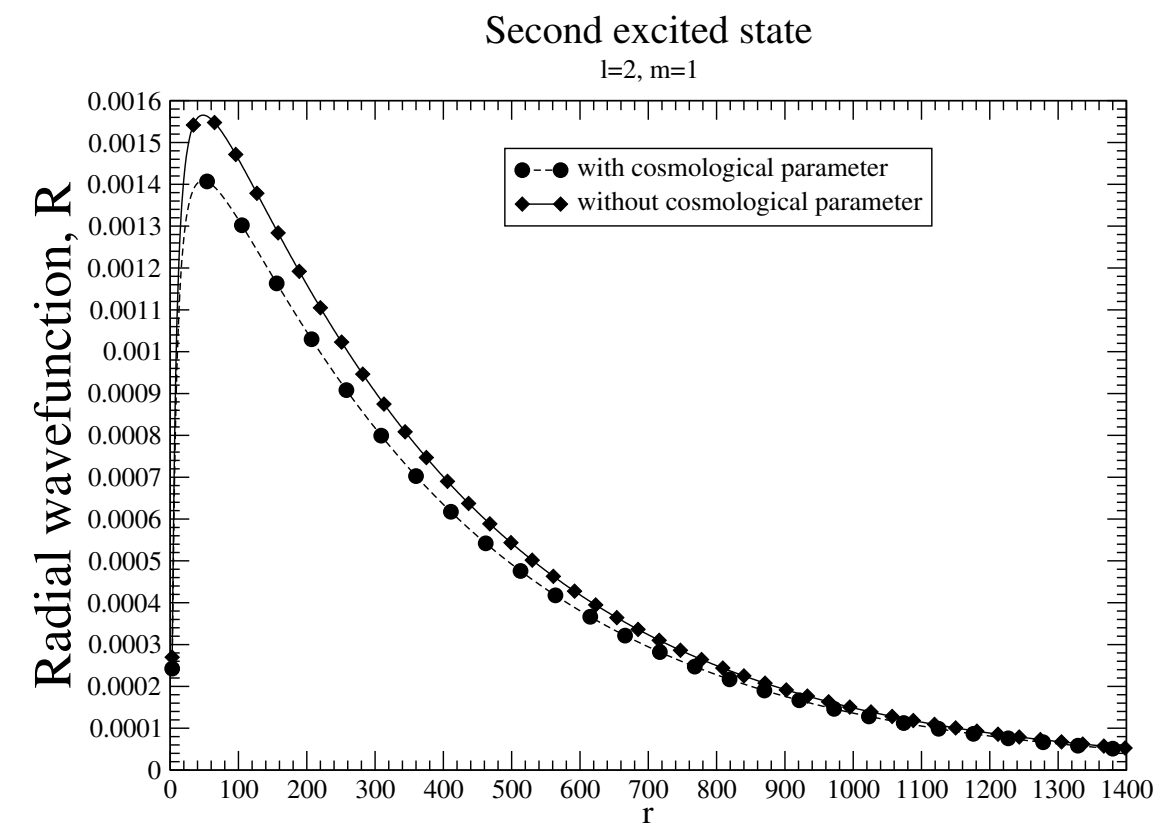

Figure 8: Radial wave function $\mathrm{R}$ of second excited state without and with cosmological constant for $\mathrm{l}=2, \mathrm{~m}=1$

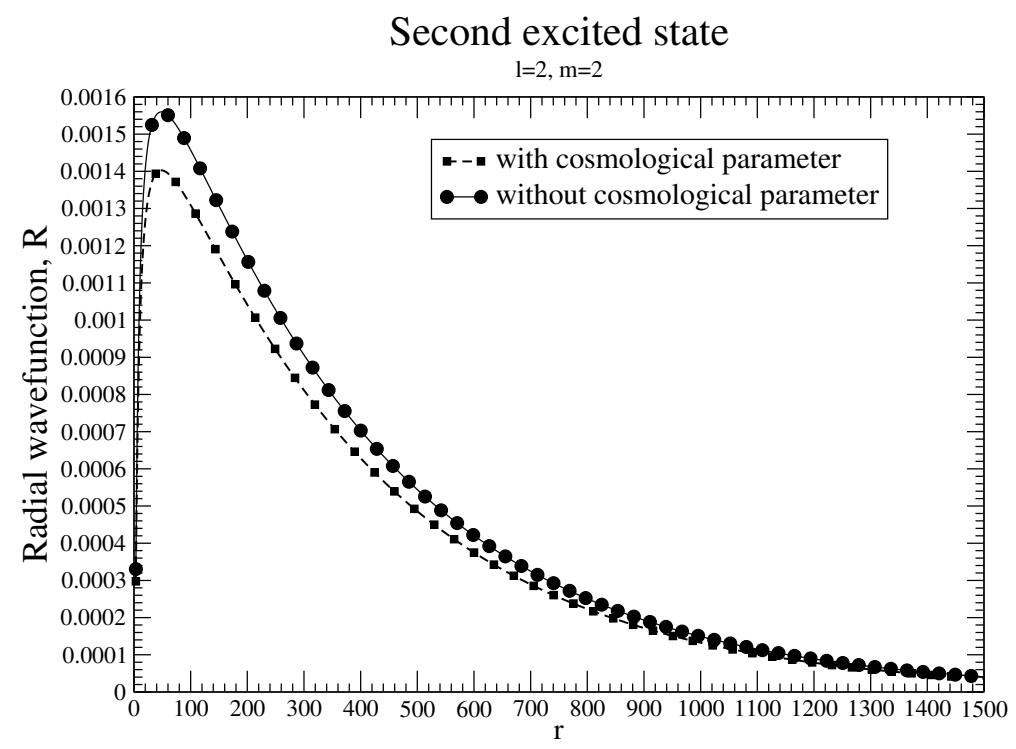

Figure 9: Radial wave function $\mathrm{R}$ of second excited state without and with cosmological constant for $1=2, m=2$ 
The next two figures 4 and 5 show the comparison of radial functions of first excited state with the inclusion of $\Lambda$ and without $\Lambda$. Similar pattern is obtained for radial wave function of first excited state in terms of decrease in the amplitude of cosmologically affected radial wave function. In Fig. 4 the radial wave function of degeneracy state $1=1$ and $m=0$ attains maximum value around 0.0058 without the cosmological constant whereas radial wave function has maximum value at 0.0054 with cosmological constant $\Lambda$. It indicates that all this graphs show the decrease in amplitude of oscillation of radial wave $R$ and potential functions while it has the $\Lambda$ value. Similarly in Fig. 5 the radial wave function has depressed amplitude with cosmological parameter and it is found that the degeneracy $m=0$ and $\mathrm{l}=1$ has higher amplitude than degeneracy $m=1$ and $l=1$ for both radial and potential functions.

In Fig.6 it has compared the amplitudes of second excited states with the effect of cosmological constant on radial wave function. The amplitude behavior of second excited state follows the same pattern as that of first excited state. This implies that for all degeneracy $\mathrm{m}$, the amplitude corresponding to cosmological constant have the lower value in comparison with the amplitude of all degeneracy $m$ corresponding to the amplitude without the cosmological constant. Now in Fig.7, 8 and 9 we plot the graphs for different degeneracy $m=0,1,2$ with and without cosmological constant $\Lambda$. The similar behaviours are obtained in these degeneracies of second excited states also. The degeneracy state i.e. $l=2, m=0$ has higher amplitude than the degeneracy state i.e. $l=2, m=1,2$. It manifest exactly the comparative presentations of lower and higher degeneracy of second excited state with the inclusion of $\Lambda$ in Lagrangian density. This type of similar pattern is obtained for radial wave function as well as potential which is probably done by the presence of unknown objects like dark matter and other astronomical objects in the present universe. So studying the rotation of BS shows the indication that in the present universe, thus a formation of quark matter during the time of universe evolution.

The value of cosmological constant $\Lambda$ use is equal to 0.020 . The ground state energy is found to be 0.19 while for first excited state it is 0.0078 and for second excited state is found to be 0.0019 . For both ground and excited states the values of energies is found to be increased after the introduction of $\Lambda$ in the Lagrangian density.

\section{References}

[1] A. G. Riess et al., 1998 Observational Evidence from Supernovae for an Accelerating Universe and a Cosmological Constant Astron. J. 1161009

[2] S. Perlmutter,G. Aldering, G. Goldhaber, R. A. Knop, P. Nugent, P. G. Castro1, S. Deustua, S. Fabbro, A. Goobar, D. E. Groom, “Astron. J.”, Vol. 517, Issue 2, pp. 565-586, 1999

[3] A. Einstein, The Special and General Theory, Henry Hold and Company, New York, 1920.

[4] E. Hubble, "Proc. Natl. Acad. Sci. U.S.A.” Vol.15, Issue 3, pp. 168-173, 1929

[5] C. Palenzuela, L. Lehner and S. L. Liebling, “Phys. Rev. D” Vol. 77, Issue 4, pp. 044036 , 2008

[6] J. R. Oppenheimer and G. M. Volkoff, “Phys. Rev.” Vol. 55, Issue 5, pp. 374-381, 1939

[7] M. S. Madsen and A. R. Liddle, "Phys. Lett. B“, Vol. 251, pp. 507-510, 1990

[8] F.E. Schunck and E. W. Mielke, "Classical and Quantum Gravity" Vol. 20, pp. R301R356, 2003

[9] G. H. Bordbar, S. H. Hendi, B. Eslam Panah, “Eur. Phys. J. Plus.” Vol. 131, Issue 5, pp. 315, 2016

[10] A. G. Riess, “Astron. J.” Vol. 116, pp. 1009-1038, 1998 
[11] Schutz B F Gravity from the Ground Up 3rd edn (Cambridge: Cambridge University Press) $p 143$

[12] Sharma R, Karmakar S and Mukherjee 2008 S Boson star and dark matter arXiv:0812.3470

[13] Schunck F E and Liddle A R 1998 Boson stars in centre of galaxies? Lect. Notes Phys. 514285

[14] D. F. Torres, S. Capozziello and G. Lambiase “Phys. Rev. D” Vol. 62, Issue 10, pp. 104012-104027, 2000

[15] B. Kleihaus, J. Kunz, C. Lammerzahl and M. List “Phys. Lett. B” Vol. 675, Issue 1, pp. 102-109, 2009

[16] B. Kleihaus, J. Kunz, C. Lammerzahl and M. List “Phys. Rev. D” Vol. 82, Issue 10, pp. 104050, 2010

[17] Hartmann B, Kleihaus B, Kunz J and Schaffer I 2013 Compact (A)dS boson stars and shells "Phys. Rev. D" Vol. 88 Issue 12, pp. 124033, 2012

[18] B. Hartmann, B. Kleihaus, J. Kunz and I. Schaffer "Phys. Lett. B" Vol. 714, Issue 1, pp. 120-126, 2012

[19] D. Astefanesei and E. Radu “Nucl. Phys. B” Vol. 665, pp. 594-622, 2003

[20] H. Arodz and J. Lis “Phys. Rev. D” Vol. 77, Issue 10, pp. 107702-107705, 2008

[21] P. Jetzer “Phys. Rept.” Vol. 220, Issue 4, pp. 163-227, 1992

[22] A. Prikas "Gen. Rel. Grav. "Vol. 36, pp.1841-1869, 2004

[23] Van der Bij, J. Jochum and M. Gleiser “Phys. Lett. B” Vol. 194, Issue 4, pp. 482-486, 1987

[24] V. Silveria, C. M. G. De-Sousa, “Phys. Rev. D” Vol. 53, Issue 10, pp.5724-5728, 1995

[25] B. Jarwal and S. S. Singh, “Mod. Phys. Lett. A” Vol. 32, Issue 9, pp. 1750037-1 175003711, 2017

[26] B. Jarwal and S. S. Singh,2017 Boson star under cornell potential Procd. of the DAE Symp. on Nucl. Phys. 62 E22

[27] S. Weinberg, Gravitation and Cosmology (Wiley, New York, 1972)

[28] R. Ruffini and S. Bonazolla, 1969 Systems of Self-Gravitating Particles in General Relativity and the Concept of an Equation of State Phys. Rev. 1871786

[29] S. Kumar, U. Kulshreshtha and D. S.Kulshreshtha, 2014 Boson stars in a theory of complex scalar fields coupled to the U(1) gauge field and gravity Classical and Quantum Gravity31 167001

[30] B. Jarwal and S. S. Singh, "Rotating boson star under weal gravity potential", XXII DAE High Energy Physics Symposium, Springer Proceedings in Physics, Delhi, India 203, pp. 789, 2018 